MATHEMATICS OF COMPUTATION

Volume 76, Number 259, July 2007, Pages 1357-1372

S 0025-5718(07)01974-6

Article electronically published on February 16, 2007

\title{
CUBATURE FORMULAS FOR SYMMETRIC MEASURES IN HIGHER DIMENSIONS WITH FEW POINTS
}

\author{
AICKE HINRICHS AND ERICH NOVAK
}

\begin{abstract}
We study cubature formulas for $d$-dimensional integrals with an arbitrary symmetric weight function of product form. We present a construction that yields a high polynomial exactness: for fixed degree $\ell=5$ or $\ell=7$ and large dimension $d$ the number of knots is only slightly larger than the lower bound of Möller and much smaller compared to the known constructions.

We also show, for any odd degree $\ell=2 k+1$, that the minimal number of points is almost independent of the weight function. This is also true for the integration over the (Euclidean) sphere.
\end{abstract}

\section{INTRODUCTION}

Let us start with a special case of our results: We find cubature formulas with

$$
N(5, d, 1)=d^{2}+7 d+1, \quad \text { and } \quad N(7, d, 1)=\left(d^{3}+21 d^{2}+20 d+3\right) / 3
$$

points such that the integral

$$
I_{d}(f)=\int_{[-1,1]^{d}} f(\mathbf{x}) d \mathbf{x}
$$

is exactly computed for all polynomials of degree at most 5 or 7 , respectively. This improves the known cubature formulas for degree 5 and $d \geq 8$ and for degree 7 with $d \geq 10$. The lower bound of Möller (1979) takes the form

$$
N_{\min }(5, d, 1) \geq d^{2}+d+1 \quad \text { and } \quad N_{\min }(7, d, 1) \geq\left(d^{3}+3 d^{2}+8 d\right) / 3 .
$$

Hence, for our method, we obtain

$$
N(5, d, 1) \approx N_{\min }(5, d, 1) \quad \text { and } \quad N(7, d, 1) \approx N_{\min }(7, d, 1) .
$$

We use $\approx$ to denote the strong equivalence of sequences, i.e.,

$$
v_{n} \approx w_{n} \quad \text { iff } \quad \lim _{n \rightarrow \infty} v_{n} / w_{n}=1 .
$$

The best results (for large $d$ ) from the literature, see Stroud (1971) and the online tables of Cools, see Cools (2003), are given by

$$
N_{\text {old }}(5, d, 1)=2 d^{2}+1 \quad \text { and } \quad N_{\text {old }}(7, d, 1)=\left(4 d^{3}-6 d^{2}+14 d+3\right) / 3 .
$$

Received by the editor August 25, 2005 and, in revised form, June 16, 2006.

2000 Mathematics Subject Classification. Primary 65D32.

Key words and phrases. Cubature formulas, Möller bound, Smolyak method, polynomial exactness.

Research of the first author was supported by the DFG Emmy-Noether grant Hi 584/2-4.

(C)2007 American Mathematical Society

Reverts to public domain 28 years from publication 
More generally, we study cubature formulas

$$
Q_{n}(f)=\sum_{i=1}^{n} a_{i} f\left(\mathbf{x}_{i}\right), \quad a_{i} \in \mathbb{R}, \mathbf{x}_{i} \in \Omega,
$$

for $d$-dimensional integrals

$$
I_{d}^{\varrho}(f)=\int_{\Omega} f(\mathbf{x}) \varrho(\mathbf{x}) d \mathbf{x} .
$$

Concerning the integral we always assume

$$
\Omega=\Omega_{1} \times \cdots \times \Omega_{d}
$$

with symmetric (and possibly unbounded) intervals $\Omega_{j} \subset \mathbb{R}$ and the product form

$$
\varrho(\mathbf{x})=\varrho_{1}\left(x_{1}\right) \ldots \varrho_{d}\left(x_{d}\right)
$$

of the weight function $\varrho$. We assume that the $\varrho_{i}$ are symmetric,

$$
\varrho_{i}(x)=\varrho_{i}(-x)
$$

with $\varrho_{i} \geq 0$ and integrability of all polynomials, although these assumptions can be relaxed. Some of our results can be slightly improved in the fully symmetric case where, in addition, all the $\varrho_{i}$ coincide.

Let $\mathbb{P}(\ell, d)$ be the space of all polynomials in $d$ variables of (total) degree at most $\ell$. A cubature formula $Q_{n}$ has a degree $\ell$ of exactness if

$$
Q_{n}(f)=I_{d}^{\varrho}(f), \quad \forall f \in \mathbb{P}(\ell, d) .
$$

We define

$$
N_{\min }(\ell, d, \varrho)
$$

to be the minimal number $n$ of knots needed by any cubature formula $Q_{n}$ of degree $\ell$ of exactness.

The numbers $N_{\min }(\ell, d, \varrho)$ and corresponding cubature formulas are only known in exceptional cases, see, e.g., Schmid (1983), Berens. Schmid and Xu (1995), and Cools (1997). Thus one is interested in upper and lower bounds for this quantity.

One is often interested in cubature formulas with knots inside the domain and positive weights. While $\mathbf{x}_{i} \in \Omega$ can always be satisfied by our method, we usually have positive and negative weights. Actually we request $\mathbf{x}_{i} \in \Omega$, see (4), although the lower bound of Möller also holds without this assumption.

\section{Problem, main Results, and conjecture}

The lower bound of Möller (1979) for centrally symmetric weight functions is the following: If $k$ is odd, then

$$
N_{\min }(2 k+1, d, \varrho) \geq 2 \operatorname{dim} \mathbb{P}_{e}(k, d)=\left(\begin{array}{c}
d+k \\
d
\end{array}\right)+\sum_{s=1}^{d-1} 2^{s-d}\left(\begin{array}{c}
s+k \\
s
\end{array}\right) .
$$

If $k$ is even, then

$$
N_{\min }(2 k+1, d, \varrho) \geq 2 \operatorname{dim} \mathbb{P}_{o}(k, d)-1=\left(\begin{array}{c}
d+k \\
d
\end{array}\right)+\sum_{s=1}^{d-1}\left(1-2^{s-d}\right)\left(\begin{array}{c}
s+k-1 \\
s
\end{array}\right) .
$$


Here $\mathbb{P}_{e}(k, d)$ denotes the subspace of $\mathbb{P}(k, d)$ generated by even polynomials and $\mathbb{P}_{o}(k, d)$ is the subspace generated by odd polynomials. We obtain (11) as special cases and for large $d$ the lower bounds are of the order

$$
\approx \frac{2 d^{k}}{k !} \text {. }
$$

See the book Mvsovskikh (1981) or Cools (1997) and, for the explicit formula, Lu and Darmofal (2004).

The best upper bounds were of the form

$$
\approx \frac{2^{k} d^{k}}{k !} \text {. }
$$

They can be proved with "fully symmetric formulas" (if the $\varrho_{i}$ are equal) or (in the general case) with the "Smolyak method" or with "sparse grids". All these notions are very much related; see Section 3 . Even for special weight functions $\varrho$ and/or for special $\ell=2 k+1$ better bounds were not known. Hence there is a gap between the lower and the upper bound of a factor of $2^{k-1}$, and we only knew (before we wrote this paper) of one exception: For the weight function

$$
\varrho(\mathbf{x})=\exp \left(-\|\mathbf{x}\|_{2}^{2}\right)
$$

it is known for $\ell=5$ that

$$
d^{2}+3 d+3
$$

function values are enough; see $\mathrm{Lu}$ and Darmofal (2004).

Observe that the weight function (7) is invariant with respect to rotations. Hence one might ask whether a result similar to (8) holds for all symmetric weight functions. We conjecture that

$$
N_{\min }(2 k+1, d, \varrho) \approx \frac{2 d^{k}}{k !}
$$

holds for all $\varrho$ and all $k$, hence the Möller bound is almost optimal. In this paper we prove this conjecture for $k=2$ and $k=3$; see Theorem 1 for more details. We also prove that the numbers $N_{\min }(2 k+1, d, \varrho)$ only mildly depend on the weight function $\varrho$; see Theorem 2 for the details.

\section{Some facts about the Smolyak method}

We study a special case of the Smolyak method, as we need it in the following. We also present methods with the upper bound (6), since they are used (twice) for our new algorithm with the improved bound. We believe that this proof technique can be used to establish the conjecture (9) in full generality. Everything in this section is known or is a minor modification of known results; see Novak and Ritter (1999).

We construct cubature formulas to compute the integral (5) as follows. First we select quadrature formulas $U_{j}^{1}, U_{j}^{2}, \ldots$ to compute the one-dimensional integrals

$$
\int_{\Omega_{j}} f(x) \varrho_{j}(x) d x .
$$

These formulas should have the following properties: The formula $U_{j}^{i}$ is exact for all univariate polynomials of degree $m_{i}$, where

$$
m_{i} \geq 2 i-1 \text {. }
$$


The formula $U_{j}^{i}$ uses the knots $X_{j}^{i}$, and the number $n_{i}=\left|X_{j}^{i}\right|$ of knots satisfies

$$
n_{i} \leq 2 i-1 \text {. }
$$

We also assume that the $X_{j}^{i}$ are symmetric and "embedded" or "nested", i.e.,

$$
X_{j}^{i-1} \subset X_{j}^{i} \quad \text { for every } i \text { and } j .
$$

By (10) and (11) the weights of $U_{j}^{i}$ are uniquely determined by its knots. Formulas with this property are often called interpolatory quadrature formulas. For simplicity we assume in this paper that the numbers $m_{i}$ and $n_{i}$ do not depend on the coordinate $j$. The formula $U_{j}^{i}$, however, may depend on $j$.

A product formula $U_{1}^{i_{1}} \otimes \cdots \otimes U_{d}^{i_{d}}$ needs $n_{i_{1}} \ldots n_{i_{d}}$ function values, sampled on a grid. The Smolyak formulas $A(q, d)$ are linear combinations of product formulas with the following key properties. Only products with a relatively small number of knots are used, and the linear combination is chosen in such a way that the interpolation property for $d=1$ is preserved for $d>1$. The formula $A(q, d)$ is defined by

$$
A(q, d)=\sum_{q-d+1 \leq|\mathbf{i}| \leq q}(-1)^{q-|\mathbf{i}|} \cdot\left(\begin{array}{c}
d-1 \\
q-|\mathbf{i}|
\end{array}\right) \cdot\left(U_{1}^{i_{1}} \otimes \cdots \otimes U_{d}^{i_{d}}\right),
$$

where $q \geq d, \mathbf{i} \in \mathbb{N}^{d}$, and $|\mathbf{i}|=i_{1}+\cdots+i_{d}$.

The cubature formula $A(q, d)$ is based on the sparse grid

$$
H(q, d)=\bigcup_{|\mathbf{i}|=q} X_{1}^{i_{1}} \times \cdots \times X_{d}^{i_{d}}
$$

we use

$$
n=n(q, d)
$$

to denote the cardinality of $H(q, d) 11$ In particular we have $n(q, 1)=n_{q}$ and we put $n(0,1)=n_{0}=0$. The recursion formula

$$
n(q+1, d+1)=\sum_{s=1}^{q-d+1} n(q+1-s, d) \cdot\left(n_{s}-n_{s-1}\right)
$$

for $n(q, d)$ is known; Novak and Ritter (1999).

Remark 1. Cubature formulas with high polynomial exactness are not often used if $d$ is large, say $d>5$. One major exception is the class of fully symmetric rules for the fully symmetric case, where also

$$
\varrho_{1}=\cdots=\varrho_{d} .
$$

Fully symmetric cubature formulas were developed by Lyness $(1965 \mathrm{a}, \mathrm{b})$, McNamee and Stenger (1967), Genz (1986), Cools and Haegemans (1994), Capstick and Keister (1996), Genz and Keister (1996) and other authors. The best results with respect to polynomial exactness are obtained by Genz (1986) and Genz and Keister (1996). The fully symmetric formulas from Genz (1986) and Genz and Keister (1996) are of the Smolyak form (13). Numerical integration with the Smolyak construction was already studied in Smolvak (1963). There are many other papers on the Smolyak method. The papers Gerstner and Griebel (1998), Novak and Ritter

\footnotetext{
${ }^{1}$ Observe that some elements of the sparse grid might get a zero weight in the formula $A(q, d)$. This would decrease the number of needed function values. Hence the "actual" number of needed function values for $A(q, d)$ might be smaller than $n(q, d)$.
} 
(1999), and Petras (2003) study the polynomial exactness of $A(q, d)$. See also Novak. Ritter. Schmitt and Steinbauer (1999) and the recent survey on sparse grids by Bungartz and Griebel (2004).

The following result is well known; see Corollary 1 of Novak and Ritter (1999).

Lemma 1. Assume (10). Then $A(d+k, d)$ has (at least) a degree $\ell=2 k+1$ of exactness.

Now we present formulas for the number $n(q, d)$ of knots that are used by $A(q, d)$. We consider two cases, important for the following.

The case $n_{i}=2 i-1$. Using (14) one obtains the recursion

$$
n(q+1, d+1)=n(q, d+1)+n(q, d)+n(q-1, d)
$$

for $q \geq d$ and $n(q, 1)=2 q-1$ and $n(d, d)=1$. Table 1 consists of numbers $n(q, d)$ with minimal $q$ such that $n(q, d) \geq \ell$; these numbers are called $N(\ell, d)$.

TABLE 1. Number of knots for Smolyak's method with $n_{i}=2 i-1$

$\begin{array}{rrrrrr}\ell & N(\ell, 5) & N(\ell, 10) & N(\ell, 15) & N(\ell, 20) & N(\ell, 25) \\ 3 & 11 & 21 & 31 & 41 & 51 \\ 5 & 61 & 221 & 481 & 841 & 1301 \\ 7 & 231 & 1561 & 4991 & 11521 & 22151 \\ 9 & 681 & 8361 & 39041 & 118721 & 283401 \\ 11 & 1683 & 36365 & 246047 & 982729 & 2908411 \\ 13 & 3653 & 134245 & 1303777 & 6814249 & 24957661 \\ 15 & 7183 & 433905 & 5984767 & 40754369 & 184327311 \\ 17 & 13073 & 1256465 & 24331777 & 214828609 & 1196924561\end{array}$

Using (15) one can get an explicit formula for $n(k+d, d)$; see Novak and Ritter (1999).

Lemma 2. For every $k \in \mathbb{N}_{0}$ and $d \in \mathbb{N}$ we have

$$
n(k+d, d)=\sum_{s=0}^{\min (k, d)}\left(\begin{array}{l}
k \\
s
\end{array}\right) \cdot\left(\begin{array}{c}
k+d-s \\
k
\end{array}\right) .
$$

Remark 2. Lemma 2 immediately implies

$$
n(k+d, d) \leq\left(\begin{array}{c}
k+d \\
d
\end{array}\right) \cdot \min \left(2^{k}, 2^{d}\right) .
$$

The case $n_{i}=2 i-1$ for $i \neq 3$ and $n_{3}=3$. If we take the Gaussian formulas $U_{j}^{2}$ with 3 knots for $\varrho_{j}$, then we already have exactness 5 , and so we can take $U_{j}^{3}=U_{j}^{2}$ and still have (10). Altogether we have

$$
n_{i}=2 i-1 \text { for } i \neq 3, \quad n_{3}=3 .
$$

Observe that in this case the sets $X_{j}^{2}$ are determined by the weights $\varrho_{j}$; we cannot choose these sets. All the other sets $X_{j}^{i}$ can be chosen arbitrarily for $i>2$, but we still assume (12). Similarly as (15) we now obtain from (14) the recursion

$$
\begin{aligned}
n(q+2, d+1)= & n(q+1, d+1)+n(q+1, d)+n(q, d) \\
& -2 n(q-1, d)+4 n(q-2, d)-2 n(q-3, d) .
\end{aligned}
$$

With this simple modification we obtain the values of Table 2 . 
TABLE 2. Number of knots for method (17)

$\begin{array}{rrrrrr}\ell & N(\ell, 5) & N(\ell, 10) & N(\ell, 15) & N(\ell, 20) & N(\ell, 25) \\ 3 & 11 & 21 & 31 & 41 & 51 \\ 5 & 51 & 201 & 451 & 801 & 1251 \\ 7 & 151 & 1201 & 4151 & 10001 & 19751 \\ 9 & 401 & 5301 & 27701 & 90601 & 227001 \\ 11 & 1003 & 19505 & 146507 & 643009 & 2040011 \\ 13 & 2133 & 63805 & 655017 & 3775769 & 15056061 \\ 15 & 4223 & 188745 & 2584167 & 19111089 & 94680111 \\ 17 & 8113 & 511625 & 9224937 & 85920449 & 522028561\end{array}$

Remark 3. Later the following will be important for the two versions of Smolyak's algorithm: In the case $n_{i}=2 i-1$ we can take arbitrary symmetric sets $X_{j}^{i}$, in particular we can take

$$
X_{1}^{2}=\cdots=X_{d}^{2}
$$

We can also normalize the weights $\varrho_{j}$ in such a way that the $U_{j}^{2}$ have the form

$$
U_{j}^{2}(f)=\gamma f(-x)+\beta_{j} f(0)+\gamma f(x),
$$

where $\gamma\left(\right.$ and $x$ ) do not depend on $j$. In addition, we can choose the $X_{j}^{i}$ in such a way that $\|\mathbf{x}\|_{2} \leq \alpha$ for each $\mathbf{x} \in H(q, d)$, where $\alpha$ is the (given) radius of the domain $\Omega$ of integration. This means that each rotation maps $\mathbf{x}$ to a point in $\Omega$.

In the second case, however, we have to use the 3 Gauß-knots for $X_{j}^{2}=X_{j}^{3}$.

Remark 4. Later we project the points $H(q, d)$ of $A(q, d)$ to a sphere of fixed radius. The origin is not projected. This projection reduces the number of points; the number of projected points $n^{*}(d+k, d)$ also depends on the sets $X_{j}^{i}$. We only need the second case, where $n_{i}=2 i-1$ for $i \neq 3$ and $n_{3}=3$. In the case $k=2$ and $k=3$ one obtains

$$
n(d+2, d)=2 d^{2}+1 \quad \text { and } \quad n^{*}(d+2, d)=2 d^{2}
$$

and

$$
n(d+3, d)=\left(4 d^{3}-6 d^{2}+20 d+3\right) / 3 \quad \text { and } \quad n^{*}(d+3, d)=\left(4 d^{3}-6 d^{2}+8 d\right) / 3 .
$$

For the last formula observe that $H(d+3, d)$ contains 7 points of the form $\mathbf{x}=$ $(\alpha, 0, \ldots, 0)$ that are projected onto two different points, hence

$$
n(d+3, d)=n^{*}(d+3, d)+4 d+1 .
$$

It seems to be difficult to compute the smallest possible number $n^{*}(d+k, d)$ for general $k$, but it is clear that

$$
n(d+k, d) \geq n^{*}(d+k, d) \geq 2^{k}\left(\begin{array}{l}
d \\
k
\end{array}\right) .
$$

Hence, for large $d$, we have $n(d+k, d) \approx n^{*}(d+k, d)$. 


\section{KNOWn Results for the Lebesgue measure}

Here we explain the best known upper bounds for $N_{\min }(\ell, d, 1)$ that we found in the literature. Again we only discuss results for large $d 2$

The results for $\ell \in\{3,5,7\}$ are classical results that can be found in Stroud (1971):

$n=2 d \quad$ for the degree $\ell=3$; this bound is sharp, $N_{\min }(3, d, \varrho)=2 d$;

$n=2 d^{2}+1 \quad$ for the degree $\ell=5$;

$n=\left(4 d^{3}-6 d^{2}+14 d+3\right) / 3 \quad$ for the degree $\ell=7$.

These results can be obtained with Smolyak's method. We explain the case $\ell=7$ : First we take, as in (17), the values $n_{2}=n_{3}=3$ and $n_{4}=7$. Now observe that the 4 new points of $X_{j}^{4}$ are symmetric but otherwise arbitrary. Hence we can take (together with 0 ) the 5-point Gauß rule with degree 9 . This means that $2 d$ weights disappear, and hence $n$ is decreased by $2 d$ compared to the general situation of (17).

The best results (so far) for $\ell>7$ can be described in the following way: We again use the sequence $m_{i} \geq 2 i-1$ and so called "delayed Kronrod-Pattersonformulas". The $n_{i}$ are defined as follows: $n_{1}=1, n_{2}=n_{3}=3, n_{4}=n_{5}=n_{6}=7$, $n_{7}=\cdots=n_{12}=15, n_{13}=\cdots=n_{24}=31$ and so on. Some of these numbers are larger than $2 i-1$ and hence we can modify those $n_{i}$, used by Petras (2003), to

$$
\tilde{n}_{i}:=\min \left(n_{i}, 2 i-1\right) .
$$

In this way one obtains the values from Table 3; see Genz (1986) who obtained the same results.

TABLE 3. Known values for the Lebesgue measure

$\begin{array}{rrrrrr}\ell & N(\ell, 5) & N(\ell, 10) & N(\ell, 15) & N(\ell, 20) & N(\ell, 25) \\ 3 & 10 & 20 & 30 & 40 & 50 \\ 5 & 51 & 201 & 451 & 801 & 1251 \\ 7 & 141 & 1181 & 4121 & 9961 & 19701 \\ 9 & 391 & 5281 & 27671 & 90561 & 226951 \\ 11 & 903 & 19105 & 145607 & 641409 & 2037511 \\ 13 & 1733 & 60205 & 642417 & 3745369 & 14996061 \\ 15 & 3263 & 168825 & 2473287 & 18743249 & 93755311 \\ 17 & 5983 & 431265 & 8522247 & 82703329 & 511676911\end{array}$

Remark 5. Observe that, up to now, there is nothing better known than the fully symmetric formulas that were introduced more than 40 years ago. We do not claim that the results of Table 3 are optimal for fully symmetric (or Smolyak) rules. It was proved by Petras (2003), however, that only minor improvements are possible if one uses Smolyak formulas. The same also holds for the more general fully symmetric formulas. For fixed $\ell=2 k+1$ and large $d$, the number of points is (at least) of the order

$$
N(2 k+1, d, \varrho) \approx \frac{2^{k} d^{k}}{k !}
$$

\footnotetext{
${ }^{2}$ We illustrate this by an example. In the case $d=10$ and $\ell=13$ we will mention a method of Genz (1986) using $n=60205$ function values. In the same paper Genz presents another method using only $n=37389$. This method, however, uses more than $2^{d}$ points for general $d$ and hence is not good for "large" $d$.
} 
while the lower bound of Möller is only of the order $\frac{2 d^{k}}{k !}$. Observe that (18) holds for all the versions of Smolyak's method that we presented here.

Remark 6. By Lemma 2 we have the bound

$$
N(2 k+1, d, 1) \leq\left(\begin{array}{c}
k+d \\
d
\end{array}\right) \cdot \min \left(2^{k}, 2^{d}\right)
$$

for the Smolyak methods described here. For fixed $k$ also Kuperberg (2004) obtains a bound of the form

$$
N(2 k+1, d, 1) \leq\left(\begin{array}{c}
k+d \\
d
\end{array}\right) \cdot C_{k} .
$$

The constant $C_{k}$ is of the order $2 \cdot k^{k} \cdot k$ !, much bigger than $2^{k}$. However, Kuperberg (2004) obtains cubature formulas with positive (even equal) weights. This is a great advantage, in particular if the function values $f\left(\mathbf{x}_{i}\right)$ are given only approximately.

For a cubature formula $Q_{n}$ we define its condition number

$$
\sigma\left(Q_{n}\right)=\frac{\left\|Q_{n}\right\|_{\infty}}{\left\|I_{d}^{\varrho}\right\|_{\infty}}=\frac{\sum_{i=1}^{n}\left|a_{i}\right|}{\int_{\Omega} \varrho(\mathbf{x}) d \mathbf{x}} .
$$

A cubature formula with positive weights has condition number $\sigma\left(Q_{n}\right)=1$ if it is exact for the constant functions. The known Smolyak formulas of degree 5 and 7 have a condition number of roughly $d^{2}$ and $d^{3}$, respectively. See Remark 8 which also shows that our new formulas have roughly the same condition numbers.

\section{Cubature formulas for the SPhere And For $M_{d, k}$}

In the following we need some known results for cubature formulas for the sphere. We use these results and the Smolyak method to construct efficient cubature formulas for the linear functional

$$
M_{d, k}(f)=\sum_{\mathbf{x} \in F(d, k)} f(\mathbf{x})
$$

where

$$
\mathbf{x} \in F(d, k) \quad \Longleftrightarrow \quad x_{i} \in\{ \pm 1,0\}, \quad \sum x_{i}^{2}=k .
$$

Of course $M_{d, k}$ itself is a cubature formula using $2^{k}\left(\begin{array}{l}d \\ k\end{array}\right)$ function values, where $k \leq d$. The point is to find a cubature formula for $M_{d, k}$ that is exact for polynomials from $\mathbb{P}(2 k+1, d)$ and uses only about $2\left(\begin{array}{l}d \\ k\end{array}\right) \approx 2 d^{k} / k$ ! points, which is the order of the lower bound of Möller.

To achieve this we use two cubature formulas for the sphere that are exact for polynomials in $\mathbb{P}(2 k+1, d)$. The first formula is obtained from the Smolyak method for the Gaussian weight function (7) by projection onto the sphere of radius $\sqrt{k}$. It has the form

$$
w M_{d, k}(f)+Q_{r}(f)
$$

where $Q_{r}(f)$ is a cubature formula with $r=O\left(d^{k-1}\right)$ points and $w>0$. In particular, we can take $r \leq n^{*}(d+k, d)-2^{k}\left(\begin{array}{l}d \\ k\end{array}\right)$ with $n^{*}(d+k, d)$ from Remark 4. This leads to

$$
r \leq 2 d \quad \text { for } k=2 \quad \text { and } \quad r \leq 2 d^{2} \text { for } k=3 .
$$

This works for any degree $2 k+1$ of exactness. The second formula $\widetilde{Q}_{n}(f)$ for $k=2,3$ is taken from Mysovkikh (1968); see also Mysovskikh (1981). It uses

$$
n=d^{2}+3 d+2 \quad \text { points if } k=2 \text { and } d \geq 4
$$


and

$$
n=\left(d^{3}+9 d^{2}+14 d+6\right) / 3 \quad \text { points if } \quad k=3 \text { and } \quad d \geq 6 .
$$

It follows that the formula $w^{-1}\left(\widetilde{Q}_{n}(f)-Q_{r}(f)\right)$ is a cubature formula for $M_{d, k}$ exact for polynomials from $\mathbb{P}(2 k+1, d)$ which uses at most

$$
d^{2}+5 d+2 \quad \text { and } \quad\left(d^{3}+15 d^{2}+14 d+6\right) / 3
$$

points for $k=2$ and $k=3$, respectively.

Let us finally explain how a Smolyak formula for the Gaussian weight function leads via projection onto the sphere $R \mathbb{S}^{d-1}$ of radius $R=\sqrt{k}$ to a cubature formula of the same degree of exactness. To this end, for $r>0$, let $\omega_{r}$ be the surface measure on the sphere of radius $r$. Also let $P$ be the radial projection from $\mathbb{R}^{d} \backslash\{0\}$ onto $R \mathbb{S}^{d-1}$ given by $P \mathbf{x}=R \mathbf{x} /\|\mathbf{x}\|_{2}^{2}$. Furthermore, let

$$
Q_{n}(f)=\sum_{i=1}^{n} a_{i} f\left(\mathbf{x}_{i}\right)
$$

be an arbitrary cubature formula which is centrally symmetric. Obviously, any Smolyak formula considered above has this property. We assume that $Q_{n}$ has degree of exactness $2 k+1$ for the Gaussian weight function. Let $\mathbf{x}^{\alpha}=x_{1}^{\alpha_{1}} \ldots x_{d}^{\alpha_{d}}$ be a monomial of degree $|\alpha|=\alpha_{1}+\ldots+\alpha_{d}=2 k$. Using polar coordinates, we obtain

$$
\begin{aligned}
\int_{\mathbb{R}^{d}} \mathbf{x}^{\alpha} \exp \left(-\|\mathbf{x}\|_{2}^{2}\right) d \mathbf{x} & =\int_{0}^{\infty} \int_{r \mathbb{S}^{d-1}} \mathbf{x}^{\alpha} d \omega_{r}(\mathbf{x}) e^{-r^{2}} d r \\
& =\int_{0}^{\infty}(r / R)^{d-1+2 k} e^{-r^{2}} d r \int_{R \mathbb{S}^{d-1}} \mathbf{x}^{\alpha} d \omega_{R}(\mathbf{x}) \\
& =c(R, d, k) \int_{R \mathbb{S}^{d-1}} \mathbf{x}^{\alpha} d \omega_{R}(\mathbf{x}) .
\end{aligned}
$$

We also have

$$
Q_{n}\left(\mathbf{x}^{\alpha}\right)=\sum_{i=1}^{n} a_{i} \mathbf{x}_{i}^{\alpha}=\sum_{i=1}^{n} a_{i}\left(\left\|\mathbf{x}_{i}\right\|_{2} / R\right)^{2 k}\left(P \mathbf{x}_{i}\right)^{\alpha} .
$$

Whenever one of the points $\mathbf{x}_{i}=0$, we simply drop the corresponding term. Since

we obtain that

$$
Q_{n}\left(\mathbf{x}^{\alpha}\right)=\int_{\mathbb{R}^{d}} \mathbf{x}^{\alpha} \exp \left(-\|\mathbf{x}\|_{2}^{2}\right) d \mathbf{x},
$$

where

$$
P Q_{n}\left(\mathbf{x}^{\alpha}\right)=\int_{R \mathbb{S}^{d-1}} \mathbf{x}^{\alpha} d \omega_{R}(\mathbf{x})
$$

$$
P Q_{n}(f)=\sum_{i=1}^{n} b_{i} f\left(P \mathbf{x}_{i}\right)
$$

with

$$
b_{i}=\frac{a_{i}\left\|\mathbf{x}_{i}\right\|_{2}^{2 k}}{R^{2 k} c(R, d, k)} .
$$

So $P Q_{n}(f)$ is a cubature formula for the sphere $R \mathbb{S}^{d-1}$ which is exact for homogeneous polynomials of degree $2 k$. Since it inherits the central symmetry from $Q_{n}$, it is also exact for homogeneous polynomials of degree $2 k+1$. Since any polynomial in $\mathbb{P}(2 k+1, d)$ restricted to $R \mathbb{S}^{d-1}$ is a sum of two homogeneous polynomials of degree $2 k$ and $2 k+1$, respectively, $P Q_{n}$ is exact for all such polynomials. 
If we choose the sets $X_{j}^{2}$ in the construction of the Smolyak formula for the Gaussian measure equal, say $X_{j}^{2}=X^{2}=\{-a, 0, a\}$, then the points $\mathbf{x} \in a F(d, k)$ are present in the Smolyak formula and get equal positive weights. So the projection of this formula to the sphere $R \mathbb{S}^{d-1}$ indeed has the form (19).

Remark 7. It will be important later on that the cubature formula derived for $M_{d, k}$ uses only points on the same sphere of radius $R=\sqrt{k}$ where the points in $F(d, k)$ live.

\section{Cubature formulas For general Weight functions}

We now derive our main result which is formulated in the following theorem.

Theorem 1. Let $\Omega$ and $\varrho$ be as always and let $k=2,3$. In the case $k=2$ we assume $d \geq 4$, in the case $k=3$ we assume $d \geq 6$. Then there exists a cubature formula $Q_{n}$ for $I_{d}^{\varrho}$ with degree $2 k+1$ of exactness which uses at most

$$
d^{2}+9 d+1 \quad \text { and } \quad\left(d^{3}+33 d^{2}+14 d+3\right) / 3
$$

points for $k=2$ and $k=3$, respectively. If the one-dimensional weight functions $\varrho_{i}$ are equal (the fully symmetric case), then the number of points can be reduced to

$$
d^{2}+7 d+1 \quad \text { and } \quad\left(d^{3}+21 d^{2}+20 d+3\right) / 3
$$

for $k=2$ and $k=3$, respectively.

Proof. We start by describing how one can pass from the special cubature formulas for $M_{d, k}$ constructed in the preceding section to cubature formulas for general weight functions $\varrho$ as in the introduction. By proper scaling, we may assume that the radius of the domain $\Omega$ of integration is at least $\sqrt{k}$. First, choose a Smolyak formula $Q_{m}$ for $\varrho$ that is exact for polynomials from $\mathbb{P}(2 k+1, d)$ and satisfies $X_{1}^{2}=\ldots=X_{d}^{2}=\{-1,0,1\}$. Then $Q_{m}$ has the form

$$
Q_{m}=v M_{d, k}+Q_{s}
$$

for some $v>0$ and

$$
s=n(k+d, d)-2^{k}\left(\begin{array}{l}
d \\
k
\end{array}\right) .
$$

In general, we have to use the case where $n_{i}=2 i-1$ for all $i \geq 1$. Then we obtain

$$
s=4 d+1 \quad \text { and } \quad s=\left(18 d^{2}+3\right) / 3
$$

for $k=2$ and $k=3$, respectively. Now we replace the part $M_{d, k}$ in (23) with the formula derived in the preceding section which uses at most as many points as given in (20). By Remark 7 all points of the final cubature formula

$$
\frac{v}{w}\left(\widetilde{Q}_{n}-Q_{r}\right)+Q_{s}
$$

are in the interior of $\Omega$. This cubature formula needs at most $n+r+s$ function values. This leads to cubature formulas with

$$
d^{2}+9 d+3 \quad \text { and } \quad\left(d^{3}+33 d^{2}+14 d+9\right) / 3
$$

points for $k=2$ and $k=3$, respectively, which exceeds (21) by just two knots.

A further reduction is possible if knots of $\widetilde{Q}_{n}, Q_{r}$ and/or $Q_{s}$ coincide. We explain how this leads to the reduced number of knots in (22) in the fully symmetric case. The reduction by two knots in the general case is achieved similarly (and easier). 
To simplify notation, we denote by $M_{d, k}^{r}$ for $r>0$ the cubature formula

$$
M_{d, k}^{r}(f)=\sum_{\mathbf{x} \in F^{r}(d, k)} f(\mathbf{x})
$$

where

$$
\mathbf{x} \in F^{r}(d, k) \quad \Longleftrightarrow \quad x_{i} \in\{ \pm r, 0\}, \quad \sum x_{i}^{2}=k r^{2} .
$$

Observe that $M_{d, k}^{1}=M_{d, k}$.

Further, we need some notation for fomulas derived from the simplex. Let $S$ be a regular simplex with vertices in the unit sphere $\mathbb{S}^{d-1}$. Let $S_{d, k}^{r}$ be the cubature formula

$$
S_{d, k}^{r}(f)=\sum_{\mathbf{x} \in G^{r}(d, k)}(f(\mathbf{x})+f(-\mathbf{x}))
$$

where $G^{r}(d, k)$ is the set of all projections of the centers of the $(k-1)$-dimensional faces of $S$ onto the sphere of radius $r$. For the formulas of degree 7 we need one more cubature formula. Denote by $p_{i j}$ the $(d+1) d$ points of the form

$$
p_{i j}=\frac{1}{4} v_{i}+\frac{3}{4} v_{j}
$$

where $v_{i}$ and $v_{j}$ are different vertices of the simplex. Then let $H^{r}(d)$ be the set of all $r p_{i j} /\left\|p_{i j}\right\|$ and define the cubature formula $\widetilde{S}_{d}^{r}$ by

$$
\widetilde{S}_{d}^{r}(f)=\sum_{\mathbf{x} \in H^{r}(d)}(f(\mathbf{x})+f(-\mathbf{x})) .
$$

Finally, let $\omega_{d}$ be the surface area of $\mathbb{S}^{d-1}$.

So assume now that $\varrho_{1}=\ldots=\varrho_{d}$. We further assume without loss of generality that $\Omega \supset[-1,1]^{d}$. We treat the degree five and seven cases separately.

Degree five. The projected Smolyak formula with degree of exactness 5 for the sphere $\mathbb{S}^{d-1}$ with $d \geq 3$ needs $2 d^{2}$ points and has the form

$$
u_{1} M_{d, 1}^{1}+u_{2} M_{d, 2}^{1 / \sqrt{2}}
$$

with

$$
u_{1}=\frac{4-d}{2 d(d+2)} \omega_{d} \quad \text { and } \quad u_{2}=\frac{1}{d(d+2)} \omega_{d} .
$$

This formula can be found in Stroud (1971) or as formula 11) for the sphere in Mysovskikh (1981).

The second formula with degree of exactness 5 for the sphere $\mathbb{S}^{d-1}$ with $d \geq 4$ needs $(d+1)(d+2)$ points and has the form

$$
v_{1} S_{d, 1}^{1}+v_{2} S_{d, 2}^{1}
$$

with

$$
v_{1}=\frac{d(7-d)}{2(d+1)^{2}(d+2)} \omega_{d} \quad \text { and } \quad v_{2}=\frac{2(d-1)^{2}}{d(d+1)^{2}(d+2)} \omega_{d} .
$$

This formula can be found in Mvsovkikh (1968) or as formula 7) for the sphere in Mysovskikh (1981).

Putting (24) and (25) together gives the following formula with degree of exactness 5 for $M_{d, 2}^{1 / \sqrt{2}}$ :

$$
\frac{1}{u_{2}}\left(v_{1} S_{d, 1}^{1}+v_{2} S_{d, 2}^{1}-u_{1} M_{d, 1}^{1}\right)
$$


We also need a Smolyak type formula for the weight function $\varrho$ with degree of exactness 5 which has the form

$$
a_{1} M_{d, 2}^{1 / \sqrt{2}}+a_{2} M_{d, 1}^{1 / \sqrt{2}}+a_{3} M_{d, 1}^{\gamma}+a_{4} Q_{0},
$$

where $Q_{0}(f)=f(0)$ and $\gamma \in(0,1) \backslash\{1 / \sqrt{2}\}$. The coefficients $a_{1}, \ldots, a_{4}$ can be derived either from the Smolyak construction or from direct computation using Sobolev's theorem which tells us that our formula has the required degree of exactness if it integrates the polynomials $1, x_{1}^{2}, x_{1}^{4}, x_{1}^{2} x_{2}^{2}$ correctly. This leads to a linear system of 4 equations for $a_{1}, \ldots, a_{4}$ which has a unique solution. To minimize the number of knots we choose $\gamma=1$.

Finally, we replace $M_{d, 2}^{1 / \sqrt{2}}$ in formula (27) with the expression (26). This leads to a formula

$$
\alpha_{1}\left(S_{d, 2}^{1 / \sqrt{2}}+\frac{d^{2}(7-d)}{4(d-1)^{2}} S_{d, 1}^{1 / \sqrt{2}}\right)+\alpha_{2} M_{d, 1}^{1 / \sqrt{2}}+\alpha_{3} M_{d, 1}^{1 / 2}+\alpha_{4} Q_{0}
$$

which is exact of degree 5 for integration with respect to $\varrho$ with $d \geq 4$. The coefficients $\alpha_{1}, \ldots, \alpha_{4}$ can be directly derived using the polynomials $1, x_{1}^{2}, x_{1}^{4}, x_{1}^{2} x_{2}^{2}$. Alternatively, they are related to $a_{1}, \ldots, a_{4}$ via

$$
\alpha_{1}=\frac{2(d-1)^{2}}{(d+1)^{2}} a_{1}, \quad \alpha_{2}=a_{3}-\frac{4-d}{2} a_{1}, \quad \alpha_{3}=a_{2}, \quad \alpha_{4}=a_{4} .
$$

Observe that we have chosen our formulas so that the final number of knots is $d^{2}+7 d+3$. This can be further reduced to

$$
d^{2}+7 d+1
$$

if we choose one of the vertices of the regular simplex $S$ as the unit vector $(1,0, \ldots$, $0)$. Observe also that in the case $d=7$ the number of knots reduces even further.

Degree seven. Let us now derive a formula with degree of exactness 7, i.e., $k=3$. The projected Smolyak formula with degree of exactness 7 for the sphere $\mathbb{S}^{d-1}$ with $d \geq 3$ needs $\left(4 d^{3}-6 d^{2}+8 d\right) / 3$ points and has the form

$$
u_{1} M_{d, 1}^{1}+u_{2} M_{d, 2}^{1 / \sqrt{2}}+u_{3} M_{d, 3}^{1 / \sqrt{3}}
$$

This formula can be found in Stroud (1971) or as formula 21) for the sphere in Mysovskikh (1981).

The second formula with degree of exactness 7 for the sphere $\mathbb{S}^{d-1}$ with $d \geq 6$ needs $\left(d^{3}+9 d^{2}+14 d+6\right) / 3$ points and has the form

$$
v_{1} S_{d, 1}^{1}+v_{2} S_{d, 2}^{1}+v_{3} S_{d, 3}^{1}+v_{4} \widetilde{S}_{d}^{1}
$$

This formula can be found in Mysovkikh (1968) or as formula 13) for the sphere in Mysovskikh (1981).

Putting (29) and (30) together gives the following formula with degree of exactness 7 for $M_{d, 3}^{1 / \sqrt{3}}$ :

$$
\frac{1}{u_{3}}\left(v_{1} S_{d, 1}^{1}+v_{2} S_{d, 2}^{1}+v_{3} S_{d, 3}^{1}+v_{4} \widetilde{S}_{d}^{1}-u_{1} M_{d, 1}^{1}-u_{2} M_{d, 2}^{1 / \sqrt{2}}\right) .
$$

We also need a Smolyak type formula for the weight function $\varrho$ with degree of exactness 7 which has the form

$$
a_{1} M_{d, 3}^{1 / \sqrt{3}}+a_{2} M_{d, 2}^{1 / \sqrt{3}}+a_{3} M_{d, 1}^{1 / \sqrt{3}}+a_{4} M_{d, 2}^{\gamma_{1}}+a_{5} M_{d, 1}^{\gamma_{1}}+a_{6} M_{d, 1}^{\gamma_{2}}+a_{7} Q_{0},
$$


where $Q_{0}(f)=f(0)$ and the numbers $\gamma_{1}$ and $\gamma_{2}$ and $1 / \sqrt{3}$ are pairwise different, between 0 and 1 . To minimize the number of knots in the following we choose $\gamma_{1}=1 / \sqrt{2}$ and $\gamma_{2}=1$.

Finally, we replace $M_{d, 3}^{1 / \sqrt{3}}$ in formula (32) with the expression (31). This leads to a formula of the form

$$
\begin{aligned}
& \alpha_{1}\left(v_{1} S_{d, 1}^{1}+v_{2} S_{d, 2}^{1}+v_{3} S_{d, 3}^{1}+v_{4} \widetilde{S}_{d}^{1}\right) \\
& \quad+\alpha_{2} M_{d, 1}^{1}+\alpha_{3} M_{d, 2}^{1 / \sqrt{2}}+\alpha_{4} M_{d, 1}^{1 / \sqrt{2}}+\alpha_{5} M_{d, 2}^{1 / \sqrt{3}}+\alpha_{6} M_{d, 1}^{1 / \sqrt{3}}+\alpha_{7} Q_{0} .
\end{aligned}
$$

The constants $\alpha_{1}, \ldots, \alpha_{7}$ can be determined by using the 7 polynomials $1, x_{1}^{2}, x_{1}^{4}$, $x_{1}^{2} x_{2}^{2}, x_{1}^{2} x_{2}^{2} x_{3}^{2}, x_{1}^{4} x_{2}^{2}$ and $x_{1}^{6}$. Observe that we have chosen our formulas so that the number of knots is

$$
\left(d^{3}+21 d^{2}+20 d+9\right) / 3
$$

This can be further reduced to

$$
\left(d^{3}+21 d^{2}+20 d+3\right) / 3
$$

if we choose one of the vertices of the regular simplex $S$ as the unit vector $(1,0, \ldots$, $0)$.

Table 4 contains the number of function values for fully symmetric weight functions. Observe that for $\ell=7$ we have to assume $d \geq 6$.

TABLE 4. New values for fully symmetric weight functions

$$
\begin{array}{rrrrrrrr}
\ell & N(\ell, 5) & N(\ell, 10) & N(\ell, 15) & N(\ell, 20) & N(\ell, 25) & N(\ell, 50) & N(\ell, 100) \\
5 & 61 & 171 & 331 & 541 & 801 & 2851 & 10701 \\
7 & - & 1101 & 2801 & 5601 & 9751 & 59501 & 404001
\end{array}
$$

It is interesting to compare these values with the lower bound (1) of Möller; see Table 5 .

TABLE 5. Möller's lower bound

$\begin{array}{rrrrrrrr}\ell & N(\ell, 5) & N(\ell, 10) & N(\ell, 15) & N(\ell, 20) & N(\ell, 25) & N(\ell, 50) & N(\ell, 100) \\ 5 & 31 & 111 & 241 & 421 & 651 & 2551 & 10101 \\ 7 & 80 & 460 & 1390 & 3120 & 5900 & 44300 & 343600\end{array}$

Remark 8. For the cube $[-1,1]^{d}$ with Lebesgue measure, Tables 6 and 7 contain the coefficients $a_{i}$ and $\alpha_{i}$ in the cubature formulas (27), (28), (32). The values of $v_{1}, \ldots, v_{4}$ and $u_{1}, u_{2}, u_{3}$ for the degree 7 formula can be found in Mysovskikh (1981).

TABle 6. Coefficients for the degree 5 formulas (27) and (28)

$$
\begin{array}{ccccc}
i & 1 & 2 & 3 & 4 \\
2^{-d} a_{i} & \frac{1}{9} & \frac{22}{45}-\frac{2 d}{9} & \frac{1}{30} & \frac{2 d^{2}}{9}-\frac{37 d}{45}+1 \\
2^{-d} \alpha_{i} & \frac{2(d-1)^{2}}{9(d+1)^{2}} & \frac{d}{18}-\frac{17}{90} & \frac{22}{45}-\frac{2 d}{9} & \frac{2 d^{2}}{9}-\frac{37 d}{45}+1
\end{array}
$$


TABLE 7. Coefficients for the degree 7 formula (32)

$$
\begin{array}{ccccc}
i & 1 & 2 & 3 & 4 \\
2^{-d} a_{i} & \frac{1}{8} & \frac{7}{20}-\frac{d}{4} & \frac{23}{70}-\frac{9}{20}+\frac{d^{2}}{4} & \frac{8}{45} \\
i & 5 & 6 & 7 \\
2^{-d} a_{i} & \frac{32}{63}-\frac{16 d}{45} & \frac{1}{21} & -\frac{d^{3}}{6}+\frac{5 d^{2}}{9}-\frac{659 d}{630}+1
\end{array}
$$

Remark 9. Victoir (2004) and Kuperberg (2004) describe, in particular, methods for $\ell=5$ and positive weights. For $d=100$ Victoir has $n=4^{12}=16777216$ and this was further improved by Kuperberg to $n=65536$ points with positive weights. See the discussion in Kuperberg (2004).

For general weights the old record was 20001; see (3). Our method needs 10701 function values; the lower bound of Möller is 10101.

\section{INDEPENDENCE OF THE WEIGHT FUNCTION}

We now use the Smolyak formulas to show that, for any fixed $k$, the minimal number of knots needed by a cubature formula of degree $2 k+1$ does not essentially depend on the weight function. Since the Möller lower bound is of order $d^{k}$, the following theorem shows that the difference can only be in the lower order terms.

Theorem 2. Let $\Omega^{(j)}$ and $\varrho^{(j)}, j=1,2$, be two regions and weight functions in $\mathbb{R}^{d}$ as described in the introduction. For $k=2,3, \ldots$, define

$$
c_{k}=\frac{2^{2 k}}{(k-1) !} \text {. }
$$

Then

$$
\left|N_{\min }\left(2 k+1, d, \varrho^{(1)}\right)-N_{\min }\left(2 k+1, d, \varrho^{(2)}\right)\right| \leq c_{k} d^{k-1}
$$

for all $d \geq k$.

Proof. Without loss of generality, we assume that the cube $[-1,1]^{d}$ is contained in the interior of $\Omega^{(1)}$ and $\Omega^{(2)}$. We choose a cubature formula $Q_{n}$ for $\varrho^{(1)}$ exact for polynomials in $\mathbb{P}(2 k+1, d)$ with $n=N_{\min }\left(2 k+1, d, \varrho^{(1)}\right)$. By proper scaling if necessary we may now assume that the knots of $Q_{n}$ are in the interior of $\Omega^{(2)}$. We also choose, for $j=1,2$, Smolyak formulas

$$
Q_{m_{j}}^{S m o l}=w_{j} M_{d, k}+Q_{r_{j}}
$$

for $\varrho^{(j)}$ of degree $2 k+1$ with $w_{j}>0$. To assure their existence, we have to work with the case $n_{i}=2 i-1$ for all $i$. In this case we can also arrange that the knots of $Q_{r_{j}}$ are contained in $[-1,1]^{d}$. Then, for $d \geq k$, the estimate

$$
r_{j} \leq 2^{k}\left(\begin{array}{c}
d+k \\
k
\end{array}\right)-2^{k}\left(\begin{array}{l}
d \\
k
\end{array}\right)
$$

follows from (16). Now

$$
\frac{w_{2}}{w_{1}}\left(Q_{n}-Q_{r_{1}}\right)+Q_{r_{2}}
$$


defines a cubature rule for $\varrho^{(2)}$ exact for polynomials in $\mathbb{P}(2 k+1, d)$ with at most $n+r_{1}+r_{2}$ knots. Observe that all the knots used are in the interior of $\Omega^{(2)}$. By (33), to prove the theorem it is enough to verify the elementary inequality

$$
2^{k}\left(\begin{array}{c}
d+k \\
k
\end{array}\right)-2^{k}\left(\begin{array}{l}
d \\
k
\end{array}\right) \leq \frac{2^{2 k}}{(k-1) !} d^{k-1}
$$

for $d \geq k$, which is equivalent to

$$
(d+k)(d+k-1) \ldots(d+1)-d(d-1) \ldots(d-k+1) \leq k 2^{k} d^{k-1} .
$$

Since the left-hand side of this inequality does not exceed $(d+k)^{k}-(d-k)^{k}$, this is an immediate consequence of

$$
(d+k)^{k}-(d-k)^{k}=2 \sum_{\substack{0 \leq i \leq k \\
i \text { odd }}}\left(\begin{array}{c}
k \\
i
\end{array}\right) d^{k-i} k^{i} \leq 2 d^{k-1} k \sum_{\substack{0 \leq i \leq k \\
i \text { odd }}}\left(\begin{array}{c}
k \\
i
\end{array}\right)=k 2^{k} d^{k-1} .
$$

Remark 10. Similarly, it can be shown that

$$
\left|N_{\min }\left(2 k+1, d, \mu_{d}\right)-N_{\min }(2 k+1, d, \varrho)\right| \leq c_{k} d^{k-1},
$$

where $\mu_{d}$ is the surface measure on the sphere $\mathbb{S}^{d-1}$ and $\varrho$ is a weight function as in Theorem 2 ,

\section{ACKNOWLEDGMENT}

We thank two anonymous referees for helpful comments.

\section{REFERENCES}

Berens, H., Schmid, H. J., and Xu, Y. (1995): Multivariate Gaussian cubature formulae. Arch. Math. 64, 26-32 MR1305657 (95i:41054)

Bungartz, H.-J., Griebel, M. (2004): Sparse grids. Acta Numerica 13, 147-269. MR2249147

Capstick, S., Keister, B. D. (1996): Multidimensional quadrature algorithms at higher degree and/or dimension. J. of Computational Physics 123, 267-273 MR1372373

Cools, R. (1997):Constructing cubature formulas: the science behind the art. Acta Numerica 6, 1-54 MR1489255 (99f:65038)

Cools (2003): An encyclopedia of cubature formulas. J. Complexity 19, 445-453 MR1984127 (2004e:41031)

Cools, R. and Haegemans, A. (1994): An imbedded family of cubature formulae for $n$-dimensional product regions. J. Comput. Appl. Math. 51, 251-262 MR1290241 (95e:65020)

Genz, A. C. (1986): Fully symmetric interpolatory rules for multiple integrals. SIAM J. Numer. Anal. 23, 1273-1283 MR865956 (88b:65032)

Genz, A. C., Keister, B. D. (1996): Fully symmetric interpolatory rules for multiple integrals over infinite regions with Gaussian weight. J. Comput. Appl. Math. 71, 299-309 MR.1399898 (97b:65028)

Gerstner, T., Griebel, M. (1998): Numerical integration using sparse grids. Numer. Algorithms 18, 209-232 MR1669959 (99m:65042)

Kuperberg, G. (2004): Numerical cubature using error-correcting codes. Preprint, arXiv:math.NA/0402047 MR2231848

Lu, J., Darmofal, D. L. (2004): Higher-dimensional integration with Gaussian weight for applications in probabilistic design. SIAM J. Sci. Comput. 26, 613-624 MR2116364 (2006a:65032)

Lyness, J. N. (1965a): Symmetric integration rules for hypercubes I-III. Math. Comp. 19, 260-276, 394-407, 625-637 MR0201067 (34:952) MR0201068 (34:953) MR201069(34:954)

Lyness J. N. (1965b): Limits on the number of function evaluations required by certain highdimensional integration rules of hypercubic symmetry. Math. Comp. 19, 638-643 MR0199961 $(33: 8101)$ 
McNamee, J., Stenger, F. (1967): Construction of fully symmetric numerical integration formulas. Numer. Math. 10, 327-344 MR0219241 (36:2324)

Möller, H. M. (1979): Lower bounds for the number of nodes in cubature formulae. In: Hämmerlin, G., ed., Numerische Integration, ISNM 45, pp. 221-230. Birkhäuser, Basel MR561295 (81j:65053)

Mysovkikh, I. P. (1968): On the construction of cubature formulas with the smallest number of nodes. Soviet Math. Dokl. 9, 277-280. [Russian original: Dokl. Akad. Nauk SSSR 178, 1252-1254.] MR 0224284 (36:7328)

Mysovskikh, I. P. (1981): Interpolatory Cubature Formulas. Nauka, Moscow. [In Russian.] MR656522(83i:65025)

Novak, E., Ritter, K. (1996): High dimensional integration of smooth functions over cubes. Numer. Math. 75, 79-97 MR.1417864 (97k:65057)

Novak, E., Ritter, K. (1999): Simple cubature formulas with high polynomial exactness. Constr. Approx. 15, 499-522 MR1702807 (2000k:65050)

Novak, E., Ritter, K., Schmitt, R., Steinbauer A. (1999): On a recent interpolatory method for high dimensional integration. J. Comput. Appl. Math. 112, 215-228 MR.1728461

Petras, K. (2003): Smolyak cubature of given polynomial degree with few nodes for increasing dimension. Numer. Math. 93, 729-753 MR1961886 (2004c:65017)

Schmid, H. J. (1983): Interpolatorische Kubaturformeln. Dissertationes Mathematicae, CCXX MR735919 (85i:65034)

Smolyak, S. A. (1963): Quadrature and interpolation formulas for tensor products of certain classes of functions. Soviet Math. Dokl. 4, 240-243

Stroud, A. H. (1971): Approximate calculation of multiple integrals. Prentice-Hall, Englewood Cliffs, NJ MR0327006 (48:5348)

Victoir, N. (2004): Asymmetric cubature formulae with few points in high dimension for symmetric measures. SIAM J. Numer. Anal. 42, 209-227 MR2051063 (2005g:65044)

Mathematisches Institut, Universität Jena, Ernst-Abbe-Platz 2, D-07743 Jena, GerMANY

E-mail address: hinrichs@math.uni-jena.de

Mathematisches Institut, Universität Jena, Ernst-Abbe-Platz 2, D-07743 Jena, GerMANY

E-mail address: novak@math.uni-jena.de 